\section{(A) OPEN ACCESS}

\title{
Serum soluble interleukin 7 receptor is strongly associated with lupus nephritis in patients with systemic lupus erythematosus
}

\author{
Valérie Badot, ${ }^{1,2}$ Remco K M A C Luijten, ${ }^{3}$ Joel A van Roon, ${ }^{3}$ Geneviève Depresseux, \\ Selda Aydin, ${ }^{4}$ Benoît J Van den Eynde, ${ }^{5}$ Frédéric A Houssiau, ${ }^{1}$ Bernard R Lauwerys ${ }^{1}$
}

\begin{abstract}
- Additional material is published online only. To view please visit the journal online (http://dx.doi.org/10.1136/ annrheumdis-2012-202364).

${ }^{1}$ Pôle de Recherche en Rhumatologie, Institut de Recherche Expérimentale et Clinique, Université catholique de Louvain and Cliniques Universitaires Saint-Luc, Brussels, Belgium

${ }^{2}$ Department of Rheumatology, Centre Hospitalier Universitaire-Brugmann, Brussels, Belgium ${ }^{3}$ Department of Rheumatology and Clinical Immunology, UMC Utrecht, Utrecht, The Netherlands

${ }^{4}$ Department of Pathology, Université catholique de Louvain and Cliniques Universitaires Saint-Luc, Brussels, Belgium ${ }^{5}$ Ludwig Institute for Cancer Research, Brussels Branch \& de Duve Institute, Université catholique de Louvain Brussels, Belgium
\end{abstract}

\section{Correspondence to} Dr B R Lauwerys, Pôle de Recherche en Rhumatologie, Institut de Recherche Expérimentale et Clinique, Université catholique de

Louvain and Cliniques Universistaires Saint-Luc, Avenue Hippocrate 10, boîte B2.5390, Brussel 1200,

Belgium; Bernard.Lauwerys@ uclouvain.be

\section{VB and RKMACL contributed} equally.

Received 16 July 2012 Revised 11 November 2012 Accepted 2 December 2012 Published Online First 22 December 2012

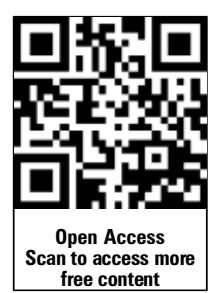

\section{ABSTRACT}

Background The soluble form of the interleukin 7 receptor (sIL-7R) is produced by fibroblasts after stimulation with proinflammatory cytokines. Increased sIL7R serum and synovial fluid levels were recently demonstrated in patients with rheumatoid arthritis. Objectives To investigate whether slL-7R production is dysregulated in systemic lupus erythematosus (SLE), and whether this correlates with disease activity.

Methods Serum and urine slL-7R concentrations were measured by ELISA, and sIL-7R quantitative PCR (qPCR) studies were performed in peripheral blood mononuclear cells (PBMCs). IL-7R, tumour necrosis factor $\alpha$ (TNF $\alpha$ ), $\mathrm{IL}-1 \beta$ and IL-17 immunostainings were performed on kidney sections.

Results slL-7R concentrations were significantly higher in SLE sera than in controls, and correlated with SLE Disease Activity Index (SLEDAI) scores. Accordingly, serum sIL-7R levels were strongly raised in patients with nephritis. Moreover in patients with lupus nephritis, serum sIL-7R decreased upon treatment. slL-7R gene expression in PBMCs was similar in patients with lupus nephritis and controls. By contrast, abundant perivascular IL-7R expression was seen in SLE kidney biopsy specimens, which was associated with expression of TNF $\alpha$ in the surrounding tissue.

Conclusions Our data indicate that SIL-7R is a marker of SLE disease activity, especially nephritis. In contrast to conventional disease activity markers, sIL-7R is not produced by immune cells, but might instead reflect activation of tissue cells in the target organ.

\section{INTRODUCTION}

Nephritis is a severe manifestation of systemic lupus erythematosus (SLE), caused in most cases by the glomerular deposition of anti-dsDNA antibodies that locally activate complement, and elicit a strong inflammatory response. Treatment of lupus nephritis (LN) usually requires the use of immunosuppressive agents in order to preserve renal function. When the disease reaches (partial or complete) remission, flares of LN can occur, requiring the administration of new cycles of treatment. ${ }^{1}$ However, detection of such flares cannot always rely on the use of systemic or renal markers of disease activity, ${ }^{2}$ and repeat biopsies are often needed in the follow-up of the patients.

The need for markers of nephritis in patients with SLE prompted us to investigate the presence of the soluble form of the interleukin 7 receptor (sIL-7R) in serum and urine samples of patients with SLE with and without nephritis. Fibroblasts and, to a lesser extent, CD4 $\mathrm{T}$ cells produce a sIL-7R, by alternative splicing of the full-length IL-7R mRNA. We recently showed that exposure of synovial fibroblasts from patients with rheumatoid arthritis to proinflammatory cytokines (tumour necrosis factor $\alpha$ (TNF $\alpha)$, IL-1 $\beta$, alone or in combination with IL-17, but not IL-6 or IL-7) induces the expression of sIL-7R. In rheumatoid arthritis, serum sIL-7R concentrations are significantly higher than in controls, and reflect disease severity and absence of response to methotrexate or TNF-blocking therapy. ${ }^{3-5}$ Given the documented presence of similar proinflammatory cytokines in the lupus kidney, ${ }^{6-9}$ we wondered whether determination of sIL-7R concentrations in serum and urine might serve as a marker of local inflammation in LN.

\section{PATIENTS AND METHODS}

Patients and controls

Serum samples were obtained from two groups of patients with SLE ( $n=27$ and 60) and age- and gender-matched healthy individuals ( $n=75$ and 44 ) in Belgium and the Netherlands. Serum sIL-7R measurements in Belgian control sera were published previously. ${ }^{4}$ All patients with SLE met the 1982 revised criteria for the classification of SLE. ${ }^{10}$ Demographic characteristics of the patients are described in table 1. All serum samples were harvested before the start of treatment in the Belgian SLE population. All patients from the Netherlands had low disease activity (SLE Disease Activity Index (SLEDAI) score between 0 and 6).

Eighteen Belgian patients with SLE had biopsyproven class III or class IV glomerulonephritis. Nine of them were included in a prospective trial $^{11}$; in these patients, follow-up serum samples were available at 3 months, after administration of low-dose intravenous cyclophosphamide $(500 \mathrm{mg}$ every 2 weeks), and corticosteroids $(0.5 \mathrm{mg} / \mathrm{kg} /$ day, progressively tapered down to $0.1 \mathrm{mg} / \mathrm{kg} /$ day at month 3 ).

Peripheral blood mononuclear cells (PBMCs) were obtained from 10 additional patients with untreated LN (median SLEDAI score: 18) and 10 controls. Spot urine samples were collected from two independent populations of patients with SLE nephritis (before or after initiation of immunosuppressive treatment) and from controls. 
Table 1 Demographic characteristics of the patients with SLE included in the serum study

\begin{tabular}{|c|c|c|}
\hline Characteristics & Belgian population $(n=27)$ & Dutch population $(\mathrm{n}=60)$ \\
\hline Women/men & $27 / 0$ & $60 / 0$ \\
\hline Arthritis, $>2$ swollen joints at baseline ( $n$ ) & 7 & 0 \\
\hline \multicolumn{3}{|l|}{ SLEDAI, median (IQR) } \\
\hline Patients without nephritis & $6(4-10)$ & $4(2-4)$ \\
\hline \multicolumn{3}{|l|}{ Serum C3 $(\mathrm{mg} / \mathrm{dl})$, mean \pm SEM } \\
\hline Patients without nephritis & $82.7 \pm 8.3$ & $90.5 \pm 3.1$ \\
\hline Patients with nephritis at baseline & $42.7 \pm 5.7$ & - \\
\hline \multicolumn{3}{|c|}{ Serum anti-dsDNA $A b$ titres by Farr assay $(\mathrm{U} / \mathrm{ml})$, mean \pm SEM } \\
\hline Non-nephritis patients & $39.8 \pm 18.6$ & $72.6 \pm 25.8$ \\
\hline
\end{tabular}

Ab, antibody; SLE, systemic lupus erythematosus; SLEDAI, SLE Disease Activity Index.

The study was approved by the ethics committees of the Université catholique de Louvain and UMC Utrecht, and informed consent was obtained from all patients.

\section{SIL-7R ELISA}

sIL-7R serum concentrations were determined by sandwich ELISA in serum and urine samples, as previously described, ${ }^{4}$ using a goat polyclonal hIL-7R antibody (Sigma-Aldrich) as coating antibody and a mouse monoclonal hIL-7R antibody as detecting antibody (Sigma-Aldrich). The sensitivity of the ELISA is $20 \mathrm{pmol} / \mathrm{ml}$.

\section{RT-qPCR experiments}

Total RNA was extracted from purified PBMCs from additional patients with untreated LN $(n=10)$, and additional controls $(\mathrm{n}=10)$ using the Nucleospin RNA II extraction kit (Macherey-Nagel), including DNase treatment of the samples. cDNA was synthesised using RevertAid Moloney murine leukaemia virus RT (Fermentas) and oligo(dT) primers. Quantitative RT-PCR was performed on a LightCycler 480 (Roche) using SYBR Green detection mix. The following $10 \mathrm{mM}$ primers were used: $\beta$-Actin: forward: GGCATCGTGAT GGACTCCG and reverse: CTGGAAGGTGGACAGCGA; sIL-7r: forward: AGCCAATGACTTTGTGGTGAC and reverse: TACGA TAGGCTTAATCCTGAG. The sIL-7R reverse primer spans the junction between exon 5 and exon 7 of the mRNA sequence of the IL-7R gene, and therefore cannot amplify the full IL-7R transcript. The melting curves obtained after each qPCR amplification confirmed the specificity of the SYBR Green assays. Normalised expression of the target genes in the studied samples was obtained using actin and sIL-7R standard curves.

\section{Immunohistochemistry on frozen sections}

Staining of frozen sections of the LN kidney $(n=6)$ or control (kidney transplant from healthy donor, $n=3$ ) biopsy samples was performed as previously described $^{3}$ with the following antibodies: interleukin 7 receptor $\alpha$ chain (IL-7R) (Sigma Aldrich, clone 40131), tumour necrosis factor $\alpha$ (R\&D Systems, clone 28401), interleukin $1 \beta$ (Lifespan Biosciences, catalogue number LS-C18827) and interleukin 17 (eBioscience, clone eBio64DEC17).

\section{Statistical analysis}

Data are expressed as mean $( \pm$ SEM) or median (range) and were analysed using SPSS V.15.0.1 and Graphpad Prism V.4.00 software. Student's t test was used to compare continuous variables with a normal distribution, and non-parametric tests (Mann-Whitney U or Wilcoxon matched-pairs signed rank tests) for categorical variables (SLEDAI) or continuous variables not distributed normally. For regression analyses, Spearman's correlation coefficients were calculated. A p value $<0.05$ was considered statistically significant.

\section{RESULTS}

First, serum sIL-7R concentrations in serum samples from patients with active SLE were measured. Patients with SLE from two different populations were characterised by significantly higher sIL-7R serum concentrations, as compared with controls ( $p<0.001$ in both groups) (figure 1A,B). Patients with active arthritis at the time of serum sampling did not display higher serum sIL-7R levels compared with patients without arthritis (data not shown). By contrast, serum sIL-7R levels were significantly higher in patients with nephritis than in patients without nephritis (figure 1B). Serum IL-7R concentrations correlated significantly with disease activity (measured by SLEDAI) in all patients (figure 1C) and also in the subgroup of patients with LN (figure 1D). In the LN group, there was no correlation between serum sIL-7R and other markers of disease activity: serum creatinine, proteinuria, serum C3 or anti-dsDNA antibody titres (data not shown). In nine patients with $\mathrm{LN}$ followed-up in a prospective trial, a strong decrease in serum sIL-7R levels was seen upon immunosuppressive treatment (figure 1E), associated with a decrease in SLEDAI scores (from 23 down to 6 median score). No significant differences in urine sIL-7R concentrations between patients with nephritis and controls were seen (see online supplementary figure S1). Taken together, these data indicate that serum sIL-7R may be a sensitive and specific marker of renal involvement in patients with SLE (figure $1 \mathrm{~F}$ ).

Next, the cellular source of sIL-7R production was investigated. qPCR experiments indicated that sIL-7R gene expression is not significantly higher in LN PBMCs than in controls (figure 2A). This suggests that other cell types, possibly at the site of inflammation, may contribute to the production of sIL-7R protein. 
A
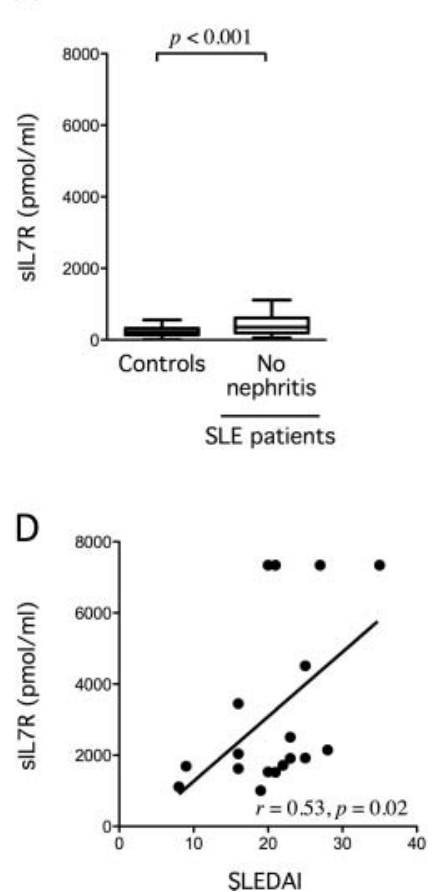

B
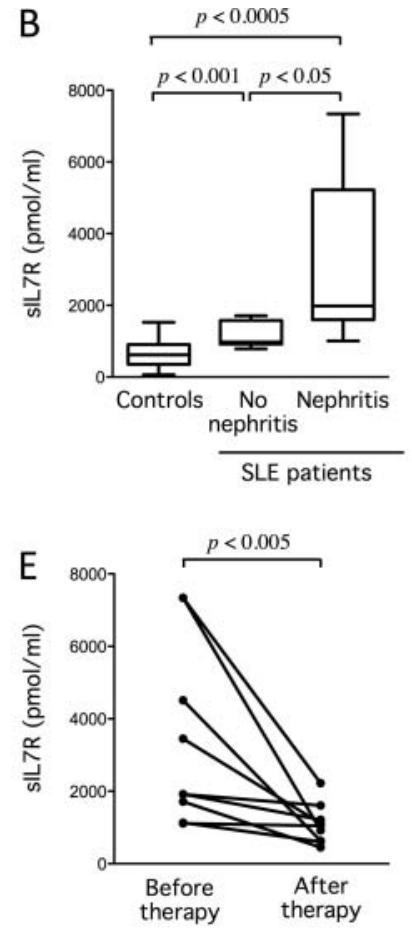

C

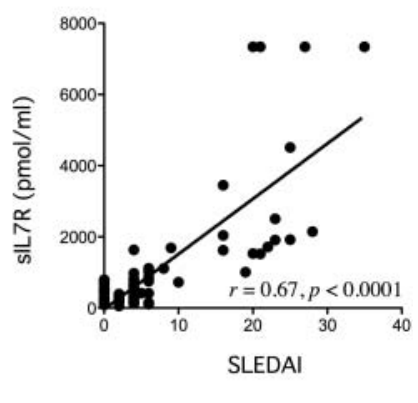

$\mathrm{F}$

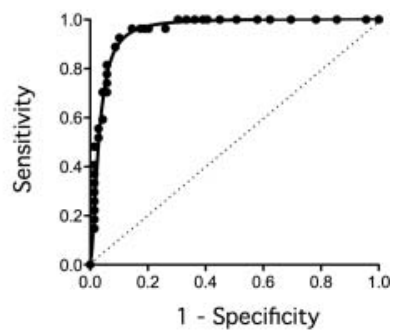

Figure 1 Strongly increased soluble form of the interleukin-7 receptor (sIL-7R) serum concentrations in lupus nephritis. slL-7R concentrations were measured by ELISA in serum samples from healthy individuals ((A) The Netherlands, $n=44,(B)$ Belgium, $n=75)$, and from patients with systemic lupus erythematosus (SLE) $((A) n=60,(B) n=27,18$ of whom had nephritis). The data are represented as the median serum concentration, interquartile range (box) and furthest value within the $1.5 \times$ interquartile range (whiskers). $p$ Values were calculated using Mann-Whitney tests. (C and D) Correlation between baseline sIL-7R serum concentrations and SLE Disease Activity Index (SLEDAI) scores in patients with SLE (C) all; (D) patients with nephritis). Spearman $r$ and $p$ values are displayed on the graph. (E) Evolution of sIL-7R concentrations between baseline and 3 months after the start of treatment with high-dose steroids and intravenous cyclophosphamide in a subset of nine patients with lupus nephritis included in a prospective trial. $\mathrm{p}$ Value by Wilcoxon matched-pair signed rank test. (F) Receiver operating characteristic analysis of serum slL-7R for the detection of nephritis in patients with SLE. The curve was plotted using sIL-7R data collected in all SLE sera, and shows the evolution of sensitivity and specificity at several serum sIL-7R thresholds.

In our previous work, we showed that sIL-7R can be produced partly by activated CD4 T cells, but mainly by activated fibroblasts, after stimulation with proinflammatory cytokines such as TNF $\alpha$, IL-1 $\beta$ and IL-17. Therefore IL-7R, TNF $\alpha$, IL-1 $\beta$ and IL-17 immunostainings were performed in kidney biopsy specimens from controls $(n=3)$ and patients with untreated LN $(\mathrm{n}=6)$. No signal was detected in any of the control biopsies (data not shown). By contrast, strong IL-7R expression was seen in perivascular fibroblast-like cells (figure 2B) in all LN samples. In the surrounding tissue, expression of TNF $\alpha$ (figure $2 \mathrm{C}$ ) but not IL-1 $\beta$ nor IL-17 (data not shown) was seen.

\section{DISCUSSION}

Our results demonstrate that sIL-7R serum concentrations are raised in patients with SLE compared with healthy controls and that they correlate with composite disease activity (SLEDAI) scores-in particular, in patients with nephritis. In addition, kidney disease suppression upon immunosuppressive treatment is associated with strong reduction in serum sIL-7R. We further provide evidence for the local production of IL-7R molecules in perivascular cells of the lupus kidney. The absence of overexpression of sIL-7R in PBMCs from patients with LN compared with controls, together with expression of IL-7R by kidney perivascular cells, suggests that raised serum sIL-7R concentrations in LN reflect activation of kidney tissue cells. The presence of TNFo in the interstitium suggests that exposure to this molecule contributes to the local production of sIL-7R, since we previously showed that sIL-7R production is induced in fibroblasts upon exposure to proinflammatory cytokines.

Variations in biological indices of systemic disease activity (serum anti-dsDNA antibody titres, serum complement) do not accurately reflect the activity of SLE. Although they have a high predictive negative value, it is well documented that rises in anti-dsDNA antibody titres, or drops in serum complement fractions are not necessarily associated with current or future flares of nephritis. ${ }^{2}$ On the other hand, kidney-specific measurements such as the presence of haematuria and the quantification of proteinuria are obviously associated with the presence of glomerular lesions, but in longstanding disease, the interpretation of these parameters needs to take into account the possibility that they result from glomerular damage rather than inflammation. Therefore, assessment of renal disease activity in SLE usually requires histological evaluation of repeat-biopsy specimens.

In this context, evaluation of serum sIL-7R concentrations could provide unique information about exposure of renal tissue cells to proinflammatory stimuli, and help clinicians to tailor their treatments accordingly. Additional sIL-7R serum measurements, performed in prospective cohorts of patients, will help further to confirm sIL-7R as a valid surrogate marker of SLE (kidney) disease activity.

Acknowledgements The authors are grateful to A-L Maudoux for technical assistance.

Contributors Study conception and design: VB, BRL. Acquisition of data: VB, RKMACL, SA. Analysis and interpretation of data : VB, RKMACL, JAvR, Geneviève $D D, B J V d E, F A H, B R L$. 


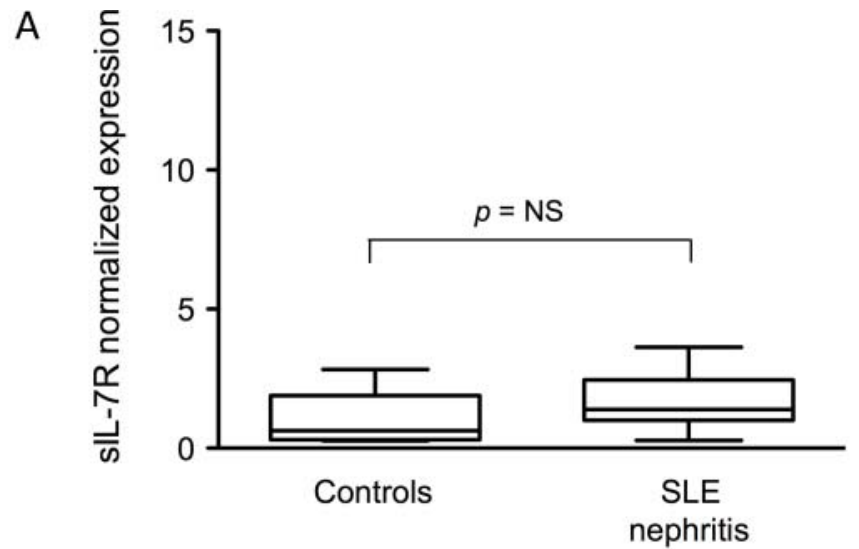

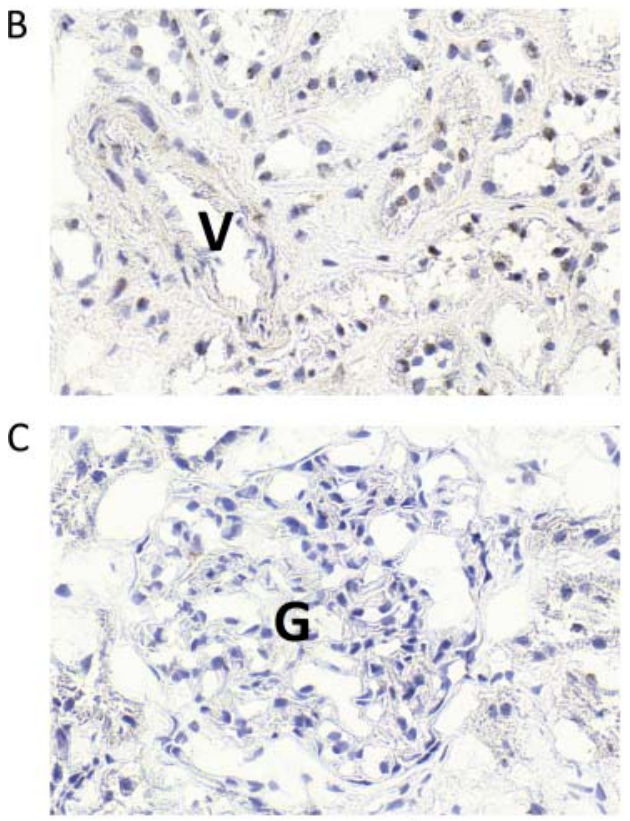

Control

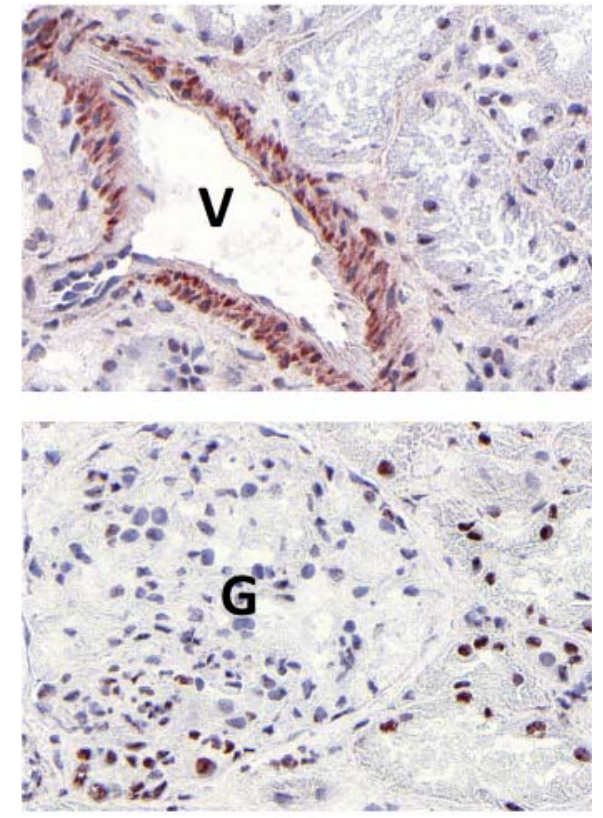

SLE

Figure 2 Expression of interleukin-7 receptor (IL-7R) and tumour necrosis factor $\alpha$ (TNF $\alpha$ ) in peripheral blood mononuclear cells (PBMCs) and kidney biopsy specimens from patients with untreated lupus nephritis.(A) Quantitative PCR evaluation of the soluble form of the interleukin-7 receptor gene expression in PBMCs from healthy individuals $(n=10)$ and from patients with untreated lupus nephritis $n=10)$. $p$ Value by Student's $t$ test. (B and C) Control ( $n=3$; left panels) and systemic lupus erythematosus (SLE; $n=6$; right panels) kidney biopsy specimens were stained with IL-7R (B) and TNF $\alpha-(C)$ specific antibodies. Typical images are shown at a $\times 400$ original magnification. $G$, glomerulus; $V$, blood vessel.

Funding This work was funded by a grant from the Fondation Saint-Luc (Cliniques Universistaires Saint-Luc, Brussels, Belgium), and a grant from BioWin (Fédération Wallonie-Bruxelles). The authors have no conflict of interest to disclose.

\section{Competing interests None.}

Provenance and peer review Not commissioned; externally peer reviewed.

Open Access This is an Open Access article distributed in accordance with the Creative Commons Attribution Non Commercial (CC BY-NC 3.0) license, which permits others to distribute, remix, adapt, build upon this work non-commercially, and license their derivative works on different terms, provided the original work is properly cited and the use is non-commercial. See: http://creativecommons.org/ licenses/by-nc/3.0/

\section{REFERENCES}

1. Houssiau FA, Vasconcelos C, D'Cruz D, et al. The 10-year follow-up data of the Euro-Lupus Nephritis Trial comparing low-dose and high-dose intravenous cyclophosphamide. Ann Rheum Dis 2010;69:61-4.

2. Bootsma H, Spronk P, Derksen $R$, et al. Prevention of relapses in systemic lupus erythematosus. Lancet 1995;345:1595-9.

3. Badot V, Galant C, Nzeusseu Toukap A, et al. Gene expression profiling in the synovium identifies a predictive signature of absence of response to adalimumab therapy in rheumatoid arthritis. Arthritis Res Ther 2009;11:R57.
4. Badot V, Durez P, Van den Eynde BJ, et al. Serum soluble interleukin-7 receptor concentrations predict response to TNF-blockade in rheumatoid arthritis. J Cell Mol Med 2011;15:2335-42.

5. Moret FM, Badot V, Lauwerys BR, et al. Characterization of IL-7 and IL-7R in the pathogenesis of rheumatoid arthritis: comment on the article by Pickens et al. Arthritis Rheum 2011:64:594-5.

6. Crispín JC, Oukka M, Bayliss G, et al. Expanded double negative T cells in patients with systemic lupus erythematosus produce IL-17 and infiltrate the kidneys. $J$ Immunol 2008:181:8761-6.

7. Yung S, Tsang RC, Sun Y, et al. Effect of human anti-DNA antibodies on proximal renal tubular epithelial cell cytokine expression: implications on tubulointerstitial inflammation in lupus nephritis. J Am Soc Nephrol 2005;16:3281-94.

8. Malide D, Russo P, Bendayan M. Presence of tumor necrosis factor alpha and interleukin-6 in renal mesangial cells of lupus nephritis patients. Hum Pathol 1995;26:558-64.

9. Aringer M, Houssiau F, Gordon C, et al. Adverse events and efficacy of TNF-alpha blockade with infliximab in patients with systemic lupus erythematosus: long-term follow-up of 13 patients. Rheumatology (Oxford) 2009;48:1451-4.

10. Tan EM, Cohen AS, Fries JF, et al. The 1982 revised criteria for the classification of systemic lupus erythematosus. Arthritis Rheum 1982;25:1271-7.

11. Houssiau FA, D'Cruz D, Sangle $S$, et al. Azathioprine versus mycophenolate mofetil for long-term immunosuppression in lupus nephritis: results from the MAINTAIN Nephritis Trial. Ann Rheum Dis 2010;69:2083-9. 